\title{
Efeitos da desnutrição precoce e reabilitação nutricional em ratos
}

\author{
Effects of early malnutrition and nutritional rehabilitation in rats
}

\author{
Magda Lahorgue Nunes ${ }^{1}$, Bianca Brum Batista ${ }^{2}$, Flávia Micheli ${ }^{3}$, Vinicius Batistella ${ }^{2}$
}

\section{Resumo}

Objetivos: verificar os efeitos da desnutrição precoce e da reabilitação nutricional em ratos em desenvolvimento, em relação ao limiar para crises convulsivas, peso corporal e peso cerebral.

Métodos: foram utilizadas ratas prenhas do tipo Wistar e suas ninhadas. Parte da ninhada foi submetida ao paradigma da desnutrição, e os animais restantes utilizados como controle. Em P15, animais desnutridos e do grupo controle foram submetidos a estado de mal convulsivo e, após recuperação das crises, iniciavam em período de reabilitação nutricional. Em P30, os ratos foram novamente submetidos a crise convulsiva induzida por flurothyl para determinação do limiar convulsivante. Após as crises, os ratos eram sacrificados, tendo o cérebro removido e pesado. A ninhada era pesada diariamente de P2 a P30.

Resultados: observamos, a partir de P5, diferença estatisticamente significativa entre o peso corporal dos animais do grupo desnutrido em comparação ao grupo nutrido. O processo de reabilitação nutricional iniciado em P16 foi associado a ascendente ganho ponderal, entretanto a diferença de peso permaneceu até $\mathrm{P} 30$. O peso cerebral foi discretamente superior em animais nutridos (média $1,47 \mathrm{~g} \pm 0,17$ ) e do sexo masculino (média $1,47 \mathrm{~g} \pm 0,16$ ), entretanto a diferença não foi estatisticamente significativa em relação aos desnutridos (média 1,42 g $\pm 0,17$ ) ou ao sexo feminino $(1,38 \mathrm{~g} \pm$ 0,12 ). O limiar (tempo em segundos) para o início da primeira crise clônica e da primeira crise tônico-clônica avaliado em P15 e P30 não apresentou diferença significativa entre os grupos.

Conclusões: nossos resultados sugerem que a desnutrição precoce não aumenta a susceptibilidade a crises convulsivas em ratos, no período de desenvolvimento, submetidos ao modelo do flurothyl. A reabilitação nutricional iniciada precocemente teria efeito protetor na susceptibilidade a crises convulsivas dos animais previamente desnutridos.

J Pediatr (Rio J) 2002; 78 (1): 39-44: epilepsia, desnutrição, recuperação nutricional.

1. Professora Adjunta de Neurologia e Pediatria da Faculdade de Medicina da PUCRS.

2. Acadêmicos da Faculdade de Medicina da PUCRS.

3. Acadêmica da Faculdade de Farmácia da PUCRS.

Artigo submetido em 27.06.01, aceito em 04.10.01.

\begin{abstract}
Objective: to verify the effects of malnutrition and nutritional rehabilitation regarding seizure threshold, body weight and brain weight in rats.

Methods: pregnant Wistar rats and their pups were used. Part of the rat pups were submitted to a malnutrition protocol and the rest served as nourished controls. At P15, malnourished and control rats were submitted to status epilepticus induced by flurothyl; and the rehabilitation period started after recovery from seizures. At P30, all rats were submitted to single flurothyl-induced seizures and the threshold was determined. After the seizures, the rats were sacrificed, the brain removed and weighed. Rat pups were weighed daily from age P2 to P30.
\end{abstract}

Results: significant differences as to body weight between malnourished and nourished rats were observed from P5 onwards. At P30, even after nutritional rehabilitation, there were still differences in terms of body weight. Nourished (mean $1.47 \mathrm{~g} \pm 0.17$ ) and male (mean $1.47 \mathrm{~g} \pm 0.16$ ) rats had brain weight slightly higher than that presented by malnourished (mean $1.42 \mathrm{~g} \pm 0.17$ ) and female (mean $1.38 \mathrm{~g} \pm 0.12$ ) rats; however, the difference was not significant. Differences observed in the threshold for the first clonic and tonic seizure at ages P15 and P30 between the groups were not statistically significant.

Conclusions: our results suggest that malnutrition does not influence seizure threshold in rat pups submitted to flurothylinduced seizures. Early nutritional rehabilitation seems to have a protective effect on seizure threshold in previously malnourished animals.

J Pediatr (Rio J) 2002; 78 (1): 39-44: epilepsy, malnutrition, nutrition rehabilitation.

\section{Introdução}

A desnutrição materno-infantil é uma das principais causas responsáveis pelo alto índice de mortalidade infantil ainda registrado no nosso país. As crianças desnutridas apresentam deficiências no seu sistema imunológico e 
maior risco de infecções, além de maior predisposição ao atraso no desenvolvimento neuropsicomotor ${ }^{1,2}$.

Dados clínicos e experimentais mostram o período neonatal como uma fase de maior susceptibilidade ao desenvolvimento de convulsões. Isso ocorre devido a uma combinação entre diversos fatores, tais como aumento da excitação e diminuição da inibição, além de alterações inerentes ao desenvolvimento em estruturas subcorticais ${ }^{3}$.

Desnutrição e epilepsia são problemas prevalentes em países em desenvolvimento, sendo uma relação causaefeito sugerida em estudos com modelo animal ${ }^{4-8}$. Até o presente momento, nenhum estudo clínico demonstrou que a desnutrição per se aumenta o risco de epilepsia, entretanto, em estudo prévio, observamos uma tendência a maior incidência de epilepsia em crianças desnutridas 9 .

Estudos experimentais evidenciam que a desnutrição perinatal causa alterações permanentes, fisiológicas e morfológicas no SNC em desenvolvimento, além de diminuir o limiar para crises convulsivas ${ }^{4-8}$. Os efeitos da desnutrição intrauterina são particularmente severos no período de proliferação e crescimento neuronal, sendo enfatizada a susceptibilidade de determinadas estruturas do sistema nervoso central, como córtex, hipocampo e cerebelo ${ }^{10,11}$. Alterações noEEG, córtex visual e sensorimotor também já foram descritas ${ }^{4-8,12}$. Deficiências específicas de nutrientes, tais como aminoácidos indispensáveis, ativam o córtex piriforme anterior, que é uma estrutura límbica bastante susceptível a crises em ratos, causando um aumento da severidade das crises e diminuição do limiar convulsivante $^{7}$.

Vários paradigmas de desnutrição são propostos na literatura ${ }^{4-8}$. Entre os mais utilizados, encontram-se a restrição alimentar pelo afastamento da mãe durante o período de lactação, dietas hipoprotéicas e dietas deficientes em aminoácidos essenciais. O modelo experimental de desnutrição crônica precoce, por restrição da ingesta alimentar ${ }^{13}$, é o mais semelhante ao que acontece na nossa população infantil, a desnutrição protéico-calórica nos dois primeiros anos de vida, iniciando após o término do aleitamento materno.

Os modelos de convulsões em animais em desenvolvimento apresentam características distintas de modelos adultos $^{14}$. A vantagem da utilização do método de inalação de flurothyl deve-se ao fato de as crises poderem ser moduladas e interrompidas rapidamente através de alterações no gotejo da droga ${ }^{13}$.

Estudos experimentais com ratos submetidos a processo de desnutrição precoce e posteriormente reabilitados do ponto de vista nutricional demonstram resultados controversos quanto à ocorrência de recuperação de estruturas do SNC previamente danificadas. O conceito de plasticidade cerebral já foi associado à recuperação do status nutricional ${ }^{15}$.

O objetivo deste estudo foi a verificação dos efeitos da desnutrição precoce e da reabilitação nutricional em rela- ção ao limiar para crises convulsivas, peso corporal e peso cerebral em ratos em desenvolvimento.

\section{Métodos}

Foram utilizadas, neste estudo, ratas prenhas do tipo Wistar e suas ninhadas. As ratas tinham livre acesso à água e ração desde o acasalamento e eram mantidas em sistema claro/escuro (12/12h), em gaiolas individuais, higienizadas, com temperatura ambiental adequada. As ninhadas foram limitadas a 10 filhotes por gaiola. A idade dos filhotes era calculada em dias de vida pós-natal, sendo o dia de nascimento da ninhada considerado como $\mathrm{P} 0$, e os dias subseqüentes como P1 a P30. Parte da ninhada era submetida ao paradigma de desnutrição e os restantes foram utilizados como controle. Em P15, animais desnutridos e do grupo controle eram submetidos a estado de mal convulsivo (ECM) e, após recuperação das crises, iniciava o período de reabilitação nutricional. Em P30, os animais eram novamente submetidos à crise convulsiva induzida por flurothyl para determinação do limiar para a primeira crise clônica e a primeira crise tônica. Após a crise, os animais eram sacrificados, o cérebro era removido e pesado. Toda a ninhada era pesada diariamente, de P2 a P30. Neste experimento, foram utilizados filhotes machos e fêmeas.

\section{Paradigma da desnutrição}

O paradigma de desnutrição utilizado consistiu na limitação da lactação através da separação da fêmea nutriz de parte da ninhada por períodos de tempo crescentes. O período de privação iniciava em $\mathrm{P} 2$ com 2 horas de afastamento e era aumentado diariamente, em 2 horas, até atingir 12 horas, ficando então fixo até P15. Durante o afastamento, os animais eram mantidos em gaiola aquecida, de forma que sua temperatura corporal se mantivesse constante (aproximadamente $34^{\circ} \mathrm{C}$ ). Após o período de privação nutricional, os filhotes retornavam para a sua gaiola com livre acesso à fêmea nutriz. Os animais controle permaneciam sempre em sua gaiola, com livre acesso à lactação ${ }^{13}$.

\section{Reabilitação nutricional}

Após recuperação do estado de mal convulsivo, os animais retornavam para a gaiola e iniciava o período de reabilitação nutricional. Os ratos tinham livre acesso ao aleitamento até P23, quando ocorria o desmame. Após o desmame, o acesso permanecia livre à água e à ração.

\section{Crises convulsivas induzidas por flurothyl}

O flurothyl [bis-(2,2,2 - trifluorethyl) éter) é um agente volátil que estimula rapidamente o sistema nervoso central, induzindo crises convulsivas generalizadas ${ }^{16,17}$. Em P15, os ratos eram testados na câmera de flurothyl, em grupos de três, sendo o gotejo mantido em 6 gotas/minuto. Cada grupo permanecia na câmera por 30 minutos, sendo o gotejo suspenso no vigésimo minuto. A primeira crise clônica e a 
primeira crise tônico-clônica de cada animal era anotada individualmente para cálculo do limiar. Posteriormente ao ECM, os ratos eram recuperados em ar ambiente, em gaiola aquecida, retornando ao restante da ninhada após às crise clínicas terem cessado totalmente. Em P30, os ratos eram submetidos, dois a dois, à nova exposição ao flurothyl. Desta vez, após a primeira crise tônico-clônica, o gotejo era suspenso e os animais removidos da câmera. A Figura 1 mostra o equipamento utilizado na aplicação da técnica do flurothyl.

Os ratos eram então sacrificados com dose elevada de barbitúrico, submetidos à perfusão transcardíaca e guilhotinados. O cérebro era removido e pesado em balança digital.

Os resultados referentes ao limiar para crises convulsivas assim como o peso dos animais e peso cerebral foram comparados através do Teste t de Student.

O estudo foi aprovado pelos Comitês Científico do HSL e de Ética da PUCRS. Os critérios éticos estabelecidos para pesquisa experimental com modelo animal foram aplica$\operatorname{dos}^{18}$.

\section{Resultados}

Foram incluídos neste estudo um total de 66 animais, machos e fêmeas, provenientes de 6 ninhadas distintas. Quarenta animais foram submetidos ao paradigma de desnutrição, e 26 serviram de controle. Durante a aplicação do paradigma de desnutrição, 18 (28\%) animais evoluíram para óbito, tendo o maior número de óbitos ocorrido em P8 (30\%). Durante o período em estado de mal convulsivo, 6 animais (3 nutridos e 3 desnutridos) evoluíram para óbito.

A aplicação do paradigma de desnutrição evidenciou, a partir de P5, diferença estatisticamente significativa entre o peso corporal dos animais do grupo desnutrido em comparação ao grupo nutrido (Figuras 2 e 3). O processo de reabilitação nutricional iniciado em P16 foi associado à curva ascendente de ganho ponderal, mas a diferença de peso entre os grupos persistiu até P30. Em ambos os grupos, os ratos machos apresentavam peso corporal (em média de 1 grama) superior ao das fêmeas do mesmo grupo. Durante o período de reabilitação nutricional foi observado que a partir de P21 as fêmeas desnutridas passavam a apresentar peso corporal igual ou superior ao dos machos desnutridos, entretanto não havia diferença significativa entre o peso corporal de machos e fêmeas dentro dos respectivos grupos de estudo entre as idades de P2 a P30. O peso cerebral verificado após perfusão intracardíaca em P30 não apresentou diferença estatisticamente significativa entre os grupos, entretanto foi discretamente superior em animais nutridos (média $1,47 \mathrm{~g} \pm 0,17$ ) e do sexo masculino (média $1,47 \mathrm{~g} \pm$ 0,16 ) em relação aos desnutridos (média $1,42 \mathrm{~g} \pm 0,17$ ) ou ao sexo feminino $(1,38 \mathrm{~g} \pm 0,12)$.

O limiar (tempo em segundos) para o início da primeira crise clônica e da primeira crise tônico-clônica foi avaliado em P15 e P30. Em P15, não ocorreu diferença estatisticamente significativa entre os grupos, entretanto foi observado que os animais nutridos apresentavam maior limiar para o desenvolvimento de crises convulsivas clônicas e tônico-

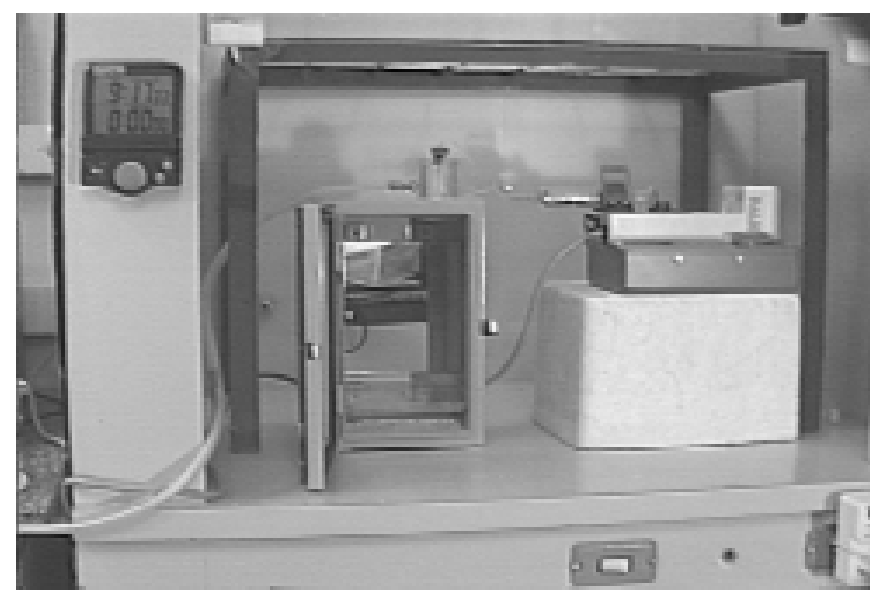

Figura 1 - Modelo de câmera vedada para experimentos com flurothyl. A bomba de infusão acoplada permite gotejo constante da droga. Na parte anterior visualiza-se dispositivo que permite a entrada de ar. A evacuação do agente é realizada através de vácuo-sucção 


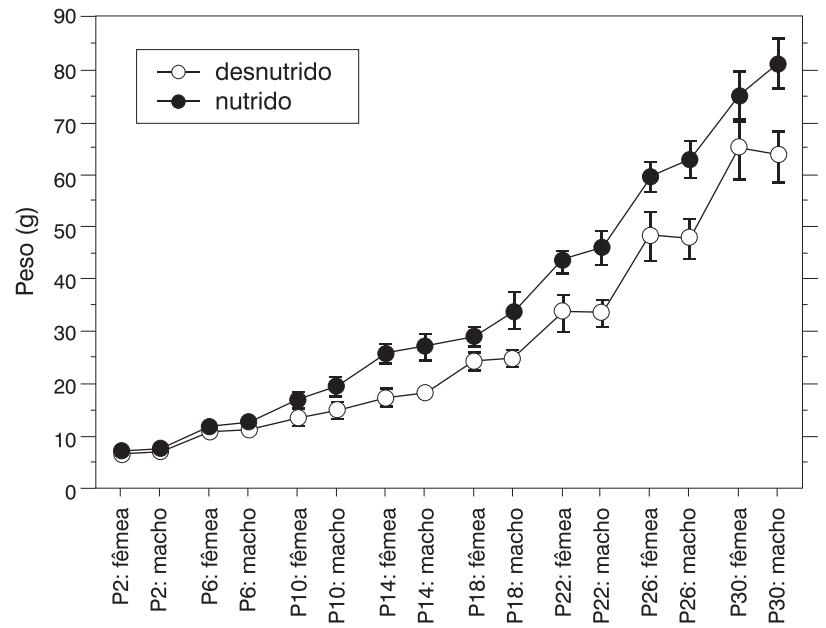

Figura 2 - O gráfico apresenta as diferenças de peso ( \pm SEM) em relação ao sexo e à idade

clônicas em comparação aos desnutridos. Tendência inversa, apesar de não estatisticamente significativa, foi observada em P30, na qual os animais previamente desnutridos apresentavam maior limiar para o desenvolvimento dos dois tipos de crises convulsivas. Não foi observada diferença significativa para o limiar de ambas as crises em relação ao sexo (Tabela 1).

Os animais apresentavam comportamento e nível de atividade semelhante durante as crises e no período de recuperação pós-ictal, independente do estado nutricional.

\section{Discussão}

Os resultados obtidos neste estudo demonstram que o paradigma de desnutrição aplicado é efetivo, pois precocemente já é possível detectar diferença significativa de peso entre os animais do grupo nutrido e desnutrido. $\mathrm{O}$ paradigma proposto, restrição de acesso ao alimento, assemelha-se ao processo de desnutrição que ocorre em famílias carentes, no qual a ingesta é inadequada, entre outras razões, pela não disponibilidade de alimentos. Os possíveis efeitos do stress nos filhotes, pela separação da mãe, foram minimizados pelo controle de condições ambientais adequadas, entretanto esse efeito certamente pode ser extrapolável à condição da desnutrição humana.

Não observamos diferenças significativas em relação ao peso corporal ou peso cerebral considerando o sexo dos animais. Em estudos prévios, tem sido utilizado de forma indistinta ratos de ambos os sexos e nenhum deles evidenciou diferenças nesse aspecto ${ }^{4,5,19,20}$.

Também não foram observadas diferenças significativas entre o peso cerebral de animais nutridos e desnutridos após reabilitação alimentar em P30. Wasterlain sugere que não é a desnutrição, mas sim a repetição de crises no cérebro, em períodos críticos do desenvolvimento, a responsável pela redução no número de células e peso cerebral $^{20}$. Em estudo prévio ${ }^{13}$, utilizando ratos desnutridos precocemente, submetidos a estado de mal convulsivo em

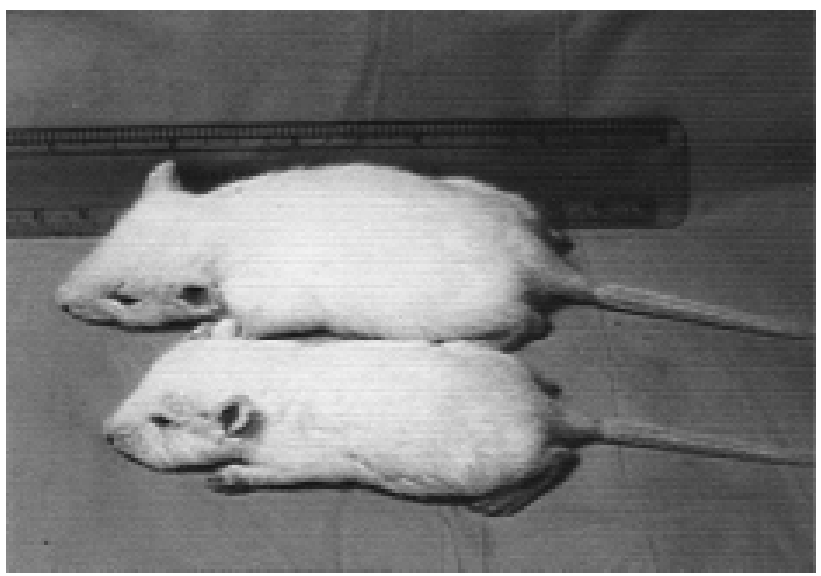

Figura 3 - A diferença de peso/tamanho dos ratos é visualmente evidente em P15

Tabela 1 - Limiar para crises convulsivas (tempo em segundos \pm dp) em P 15 e P30 em relação ao estado nutricional e ao sexo

\begin{tabular}{lcccc}
\hline Tipo de crise/ idade & Nutridos & Desnutridos & Machos & Fêmeas \\
\hline Clônica P15 & $567 \pm 152$ & $495 \pm 187$ & $538 \pm 174$ & $538 \pm 163$ \\
Tônica P15 & $614 \pm 168$ & $537 \pm 151$ & $535 \pm 113$ & $635 \pm 179$ \\
Clônica P30 & $521 \pm 70$ & $536 \pm 152$ & $554 \pm 152$ & $501 \pm 72$ \\
Tônica P30 & $660 \pm 149$ & $746 \pm 110$ & $729 \pm 127$ & $691 \pm 41$ \\
\hline
\end{tabular}

Obs.: as diferenças entre os grupos não foram significativas $(p>0,05)$ 
P15, sem reabilitação nutricional, observamos diminuição do tamanho cerebral de animais desnutridos quando comparados aos controles (observação não publicada). Os achados opostos observados em nosso primeiro estudo em P15 e no atual, em P30, podem estar relacionados tanto ao efeito das crises em período em que ocorre extenso crescimento e diferenciação celular (idade P15) como ao efeito da reabilitação nutricional.

Registramos ganho de peso constante durante a reabilitação nutricional, entretanto os ratos do grupo desnutrido permaneciam em $\mathrm{P} 30$ com peso significativamente inferior aos do grupo controle. A persistência de diferenças no peso corporal, mesmo após reabilitação alimentar, por tempo mais prolongado (até P90) foi observada também por Sharma e colaboradores ${ }^{21,22}$.

O efeito da desnutrição no limiar para crises convulsivas parece ser uma questão idade e modelo dependente. Animais que sofreram desnutrição intrauterina ou na fase de lactação submetidos a crises na idade adulta apresentam maior susceptibilidade ao desenvolvimento de convulsões ${ }^{4-8}$. Animais desnutridos submetidos aos modelos de ácido caínico e kindling também apresentam maior susceptibilidade ao desenvolvimento de crises. A formação hipocampal é uma das estruturas mais atingidas pela desnutrição, e os modelos de crise acima descritos geralmente induzem alterações permanentes em estruturas hipocampais $10,11,23$. Utilizando o modelo do flurothyl, que induz crises convulsivas de forma aguda, não observamos diferenças quanto ao limiar para crises convulsivas em nenhuma das idades avaliadas (P15 e P30), mesmo após reabilitação nutricional. Entretanto, comparativamente aos demais estudos, iniciamos a reabilitação alimentar em idade precoce, P16, sendo extrapolado para idade em humanos, equivale a 3-4 anos de vida.

Os resultados de nosso estudo sugerem que a desnutrição precoce não aumenta a susceptibilidade a crises convulsivas em ratos, no período de desenvolvimento, submetidos ao modelo de crises convulsivas induzidas por flurothyl. A reabilitação nutricional iniciada precocemente parece ter efeito protetor na susceptibilidade a crises convulsivas dos animais previamente desnutridos.

\section{Agradecimentos}

Agradecemos à Prof ${ }^{a}$ Ligia Coutinho e à Técnica Raquel Oliveira pelo auxílio prestado na perfusão intracardíaca dos animais.

Todos os acadêmicos co-autores deste artigo foram bolsistas de iniciação científica durante o desenvolvimento do projeto. BB da FAPERGS, FM e VB do programa PIBIC- PUCRS.

\section{Referências bibliográficas}

1. Tourinho H. Desnutrição na infância. In: Duncan BB, Schmidt MI, Giugliani E, eds. Medicina Ambulatorial. Porto Alegre: Editora Artes Médicas, 1992.p.85-91.

2. Grantham-Mc Gregor SM, Powell C, Stewart M, Schofield WN. Longitudinal study of growth and development of young Jamaican children recovering from severe protein-energy malnutrition. Develop Med Child Neurol 1982; 24: 321-31.

3. Moshé SL. Seizures in the developing brain. Neurology 1993; 43:S3-S7.

4. Bronzino JD, Austin-La France JR, Morgane PJ. Effects of prenatal protein malnutrition on perforant path kindling in the rat. Brain Research 1990; 515: 45-50.

5. Bronzino JD, Austin-La France RJ, Chester JS, Morgane PJ. Effect of protein malnutrition on hippocampal kindling: electrographic and behavioral measures. Brain Research 1986; 384: 348-54.

6. Taber KH, Fuller GN, Stanley JC, De France JF, Wiggins RC. The effects of postnatal undernourishment on epileptiform kindling of dorsal hippocampus. Experientia 1980; 36: 69-70.

7. Gietzen DW, Dixon KD, Truong BG, Jones AC, Barret JA, Washburn DS. Indispensable amino acid deficiency and increased seizure susceptibility in rats. AM J Physiol 1996; 271 : R1-R7.

8. Palencia G, Calvillo M, Sotelo J. Chronic malnutrition caused by a corn-based diet lowers the threshold for pentylenetetrazoleinduced seizures in rats. Epilepsia 1996; 37: 583-86.

9. Nunes ML, Teixeira GC, Fabris I, Gonçalves RA. Evaluation of the nutritional status in institutionalized children and its relationship to the development of epilepsy. Nutritional Neuroscience 1999; 2: 139-45.

10. Dobbing J. Malnutrition et développement du cerveau. La Recherche 1976; 7: 139-46.

11. Dobbing J, Hopewell JW, Lynch A. Vulnerability of developing brain. Exp Neurol 1971; 32: 439-47.

12. Salas M, Rosales A, Torrero C, Pulido S. Effects of neonatal undernutrition on the electrocortical development of the association areas in the rat. Bol Estud Méd Biol Méx 1990; 38: 3-9.

13. Nunes ML, Liptáková S, Velísková J, Sperber EF, Moshé SL. Malnutrition increases dentate granule cell proliferation in immature rats after status epilepticus. Epilepsia 2000; 41(suppl.6): S48-S52.

14. Velísek L, Velískova J, Moshé SL. Developmental seizure models. Ital J Neurol Sci 1995; 16: 127-33.

15. Schoneit B, Haensel P. Neurohistologic studies of cortical pyramidal neurons in the rat following rehabilitation of early postnatal malnutrition. J Hirnforsch 1988; 29: 599-615.

16. Truitt EB, Ebesberg EM, Ling ASG. Measurement of brain excitability by use of hexafluorodiethyl ether (Indoclon). J Pharmacol Exp Ther 1960; 129: 445-53.

17. Velísková J, Velísek L, Nunes ML, Moshé S. Developmental regulation of regional functionality of substantia nigra GABA A receptors involved in seizures. Eur J Pharmacol 1996; 309: 167-73.

18. Lopes DK, Considerações éticas e legais sobre a utilização de animais em experimentação científica. JLBE 1992; 5:19-22.

19. Wasterlain CG. Neonatal seizures and brain growth. Neuropädiatrie 1978; 9: 213-28.

20. Wasterlain CG. Developmental effects of seizures: role of malnutrition. Pediatrics 1976; 57: 197-200.

21. Sharma SK, Behari M, Maheshwari MC, Selvamurthy W. Seizure susceptibility \& intrarectal sodium valproate induced recovery in developing undernourished rats. Indian J Med Res 1990; 92: 120-27. 
22. Sharma SK, Selvamurthy W, Maheshwari MC, Singh TP. Kainic acid induced epileptogenesis in developing normal 7 undernourished rats - a computerised EEG analysis. Indian J Med Res 1990; 92: 456-66.

23. Sarkisian MR, Tandon P, Liu Z, Yang Y, Hori A, Holmes GL, et al. Multiple kainic acid seizures in the immature and adult brain: ictal manifestations and long-term effects on learning and memory. Epilepsia 1997; 38: 1157-66.
Endereço para correspondência:

Dra. Magda Lahorgue Nunes

Serviço de Neurologia do Hospital São Lucas da PUCRS

Av. Ipiranga, 6690 - sala 220

CEP 90610-000 - Porto Alegre, RS

Fone/Fax: (51) 3339.4936

E-mail:magdalahorgue@conex.com.br, nunes@pucrs.br 